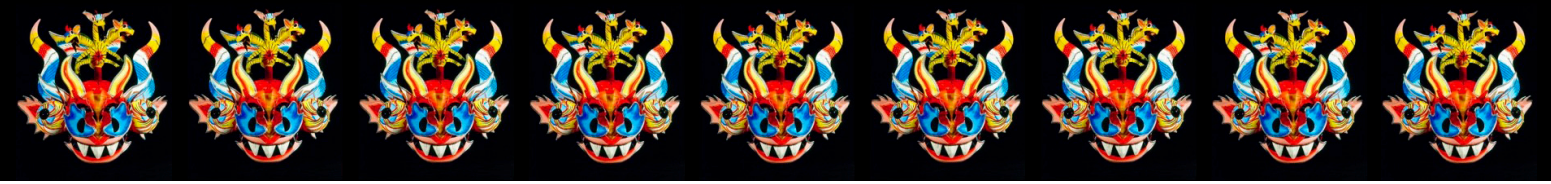

Artículo de reflexión E09A18. * Recibido: 02-12-2016. * A Aprobado en versión final: 03-01-2018. JEL: Q01. Pp. 235-255

\title{
Evaluación del estado de los Objetivos del Desarrollo del Milenio -ODM7- en el Oriente Antioqueño
}

\author{
Evaluation of state of the Millennium Development Goals \\ -MDG7- in Eastern Antioquia region.
}

Wilinthon James Osorio Vélez - Juan Felipe Cifuentes Sepúlveda

\section{COLOMBIA}

Resumen: La apuesta internacional desde las Naciones Unidas para evaluar el estado actual de los retos que sugieren una transformación de las condiciones sociales y ambientales de los países, ha permitido medir, a partir de los Objetivos de Desarrollo del Milenio (ODM) formulados en la Cumbre del Milenio en 2000, las metas para mejorar la gestión ambiental y social en el planeta. En este artículo se exploran los alcances y retrocesos de los objetivos en la región del Oriente Antioqueño y, en particular, los asuntos más relevantes para atender en las subregiones que integran dicha región. Este análisis se hace a través de la exploración documental sobre el séptimo objetivo y la información disponible sobre el tema en las alcaldías asociadas a la región.

Palabras claves: sostenibilidad ambiental; pobreza extrema y miseria; objetivos de desarrollo del milenio; oriente antioqueño.

Abstract: International commitment from United Nations to assess the current state of the challenges that suggests a transformation of the social and environmental conditions of countries, has made it possible to measure from the Millennium Development Goals (MDGs) formulated at the Millennium Summit in 2000, the goals to improve environmental and social management on the planet; It explores in this article the scope and setbacks of the objectives in the region of eastern Antioquia, in particular the most relevant issues to be addressed in the subregions that make up the territory, this analysis is done through documentary exploration of the seventh objective and the information available on the subject in the mayorships associated with the region.

Keywords: environmental sustainability; extreme poverty and misery; millennium development goals; eastern antioquia.

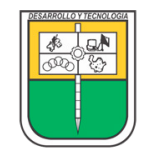

POLITÉCNICO COLOMBIANO JAIME ISAZA CADAVID
Wilinthon Osorio V. es Contador Público del Politécnico Colombiano Jaime Isaza Cadavid, Magíster en Tecnología e Ingeniería Ambiental de la Universidad Internacional Iberoamericana - Puerto Rico, docente del Politécnico Colombiano Jaime Isaza Cadavid y del Tecnológico de Antioquia - Institución Universitaria, Coordinador del semillero de investigación S.O.S Contable del Politécnico Colombiano (Sede Oriente). Contacto: wilyoso@gmail.com.

Juan Felipe Cifuentes S. es Contador Público del Politécnico Colombiano Jaime Isaza Cadavid, miembro del semillero de investigación S.O.S Contable del Politécnico Colombiano (Sede Oriente).

Contacto: juanfcifuentes-1990@hotmail.com. 


\title{
Avaliação do estado dos Objectivos do Desenvolvimento do Milénio -ODM7- na região do Antioquia Leste.
}

\begin{abstract}
Resumo: A aposta internacional desde as Nações Unidas para avaliar o estado atual dos reptos que sugere uma transformação das condições sociais e ambientais dos países, tem permitido medir desde os Objetivos de Desenvolvimento do Milénio -ODM- formulados na Cimeira do Milénio no 2000, as objetivos para melhorar a gestăo ambiental es social no planeta, Se explora no presente artigo os alcances e retrocessos de objetivos na região do oriente antioqueño, em particular os assuntos mais relevantes a atender nas subregiões que integram o território, se há este análisis através da exploração documental sobre o séptimo objetivo y a informação disponível sobre o tema em as alcaldias associadas à subregión.
\end{abstract}

Palavras-chave: sustentabilidade ambiental; pobreza extrema e miseria; metas de desenvolvimento do milenio; Antioquia Leste.

\section{Introducción}

as dificultades socioeconómicas y ambientales que se manifiestan en nuestros días están llegando a un punto crítico, en el que cada vez se hace más evidente que la tendencia de consumo del modelo capitalista está provocando la destrucción del planeta y, con ello, la de los seres que lo habitan. Las cifras de pobreza y miseria actuales son alarmantes, uno de cada cinco habitantes del mundo carece de los recursos para satisfacer las necesidades físicas y psíquicas básicas que le garantice un nivel de vida digno (PNUD, 2014:12). La crisis ecológica provocada por las actividades humanas es otro asunto inquietante a nivel mundial, el calentamiento global, la pérdida de biodiversidad, la destrucción de suelos y la aniquilación de los recursos naturales, entre otros, demuestran un panorama desolador. La Organización de las Naciones Unidas en su Programa de las Naciones Unidas para el Desarrollo ha contemplado ocho propósitos denominados Objetivos de Desarrollo del Milenio (ODM)', encaminados a superar los problemas más graves o radicales de la vida cotidiana.

La preocupación por la crisis socioeconómica y ambiental es de tal magnitud que los gobiernos nacionales y regionales han decidido incluir estrategias para la protección de los recursos naturales y políticas

1 Los Objetivos de Desarrollo del Milenio-ODM-son el resultado de la Cumbre del Milenio, organizada por la ONU y realizada en septiembre de 2000, que plantean los retos que para las naciones tiene el presente siglo y se enfocan en ocho objetivos que buscan establecer las prioridades del desarrollo en el mundo en los ámbitos económico, social y ambiental. 
fundamentadas en los principios del desarrollo sostenible en sus planes de gobierno, con el interés de evitar la irreversibilidad de la crisis ambiental y mejorar las condiciones de vida de la población.

Inicialmente el trabajo desarrolla una mirada de contexto regional en América Latina en materia de medio ambiente y desarrollo sostenible, posteriormente hace un análisis de cómo se han aplicado las metas y los instrumentos de medición de los indicadores en Colombia, una mirada general en la región del Oriente Antioqueño y, particularmente, los alcances del séptimo objetivo del milenio² en esta región.

Este artículo intenta aprovechar espacios académicos más flexibles donde se pueda demostrar que la contaduría pública puede realizar aportes en temas socioeconómicos y ambientales desde una postura interdisciplinaria, precisamente en estos tiempos donde los problemas que enfrenta la civilización contemporánea exige que se modifiquen las tendencias paradigmáticas de los campos de estudio modernos para dar un rumbo diferente a la productividad académica.

\section{Análisis de referentes}

\subsection{América Latina}

El panorama ambiental en América Latina está enfocado principalmente en la integración de los principios del desarrollo sostenible a través de políticas y programas nacionales. Algunas de estas políticas plantean principalmente la reducción en la pérdida de la biodiversidad, en razón a que esta es una de las regiones más biodiversas del mundo.

Otro aspecto importante para trabajar en la región es la reducción de los índices de pobreza y miseria, la vivienda digna para las familias, el acceso al agua potable y a los servicios básicos de saneamiento. Finalmente, el impacto del cambio climático en los distintos territorios del continente y, en particular, su efecto en los recursos hídricos.

De acuerdo con el informe de Naciones Unidas (2010), se han dado avances y retrocesos en la puesta en marcha de estos propósitos; en

2 Si bien el propósito de la presente investigación está enfocado en los alcances del séptimo Objetivo de Desarrollo del Milenio, no es posible pasar por alto el primer objetivo que tiene que ver con los niveles de erradicación de la pobreza extrema y el hambre, dado que los indicadores sobre cobertura de servicios públicos básicos y el acceso al agua potable que son metas del objetivo siete, también son determinantes en el establecimiento de las Necesidades Básicas Insatisfechas NBI, que son los estándares que determinan la pobreza y la miseria en el país. 
primer lugar, se presentan logros en la declaratoria de áreas protegidas con fines de conservación, también se presenta un aumento en el acceso al agua potable, a los servicios básicos de saneamiento y una notable disminución de la población urbana que vive en tugurios; sin embargo, las emisiones de gases contaminantes han aumentado y aún se da una creciente pérdida de cobertura de bosques en el continente por la industrialización y el capitalismo aberrante que consume los recursos naturales, deteriorando de una forma acelerada los ecosistemas.

\subsection{ODM en Colombia}

En Colombia, el compromiso hacia el logro de los ODM se materializó en el documento CONPES 91 de 2005, que luego fue ajustado en el CONPES 140 de 2011. Este último documento definió 58 indicadores a nivel nacional, de los cuales 29 son medibles a nivel departamental y a nivel municipal son 22, más 12 que pueden medirse de manera aproximada (PNUD, 2012: 8).

A continuación, se quiere precisar algunos aspectos en los alcances logrados por Colombia en la cobertura de bosque natural, el manejo de las áreas protegidas en el país, el consumo de sustancias agotadoras de la capa de ozono, el acceso al agua potable y al saneamiento básico, y finalmente información relacionada con los asentamientos precarios en las urbes colombianas.

Gráfico 1. Reforestación Plan Nacional de Desarrollo

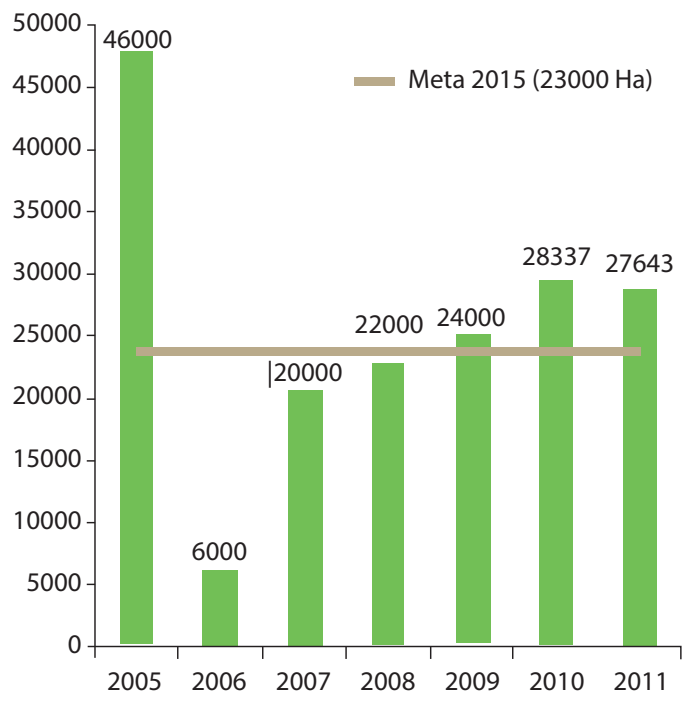

Fuente: Planeación Nacional. Informe 2009 y 2012 sobre avance de ODM en Colombia 


\section{Cobertura de bosque natural}

EI IDEAM había reportado en 2004 un área de bosque natural de 55,6 millones de hectáreas (cobertura de $48,8 \%$ de la superficie del país); sin embargo, en 2007 la estimación con base en la misma información se elevó a 61,2 millones (CEPAL, 2007: 149).

\section{Áreas protegidas por el sistema de parques}

El país ha logrado superar ampliamente y de manera anticipada la meta propuesta en este indicador. El porcentaje de ecosistemas que están incluidos y adecuadamente representados en el Sistema de Parques Nacionales Naturales es del $59 \%$, es decir, de los 144 tipos de ecosistemas existentes, 85 tienen algún tipo de representatividad en el sistema, lo anterior teniendo como referencia los ecosistemas descritos para el país en el Mapa de Ecosistemas Marinos, Costeros y Continentales (IDEAM, 2008) ${ }^{3}$.

\section{Agua potable y Saneamiento Básico}

El acceso a agua potable y saneamiento básico se ha incrementado constantemente y la meta nacional está próxima a ser cumplida. Es necesario prestar atención en las áreas rurales y garantizar el crecimiento sostenido en acceso tal y cómo se ha venido dando, con el fin de lograr el cumplimiento de la meta.

A nivel regional sobresalen el incremento del acceso a fuentes mejoradas de agua potable para la región Atlántica, pasando de 81,52\% en 2003 a $85 \%$ en 2008; le siguen la región Pacífica y Bogotá, las que presentaron incrementos por encima de la dinámica poblacional. A su vez, el acceso a servicios adecuados de saneamiento presentó un incremento considerable en la región Atlántica en donde pasó de 62,1\% en 2003 a $70,4 \%$ en 2008 , le siguen la región Pacífica y Oriental con un incremento de 3,4 porcentuales, cada una en el mismo periodo (CEPAL, 2007: 164).

\section{Asentamientos precarios}

Un HÁBITAT recomienda tener en cuenta cinco dimensiones para la definición del Indicador de Asentamientos Precarios - IAP:

1. Acceso a agua potable.

2. Acceso a saneamiento básico. 
3. Tenencia segura: es el derecho a contar con una protección eficaz del Estado contra los desalojos forzados.

4. Durabilidad de la vivienda.

5. Hacinamiento: área suficiente para vivir.

En cuanto a la evolución de los asentamientos precarios, entre 2003 y 2008 se destaca la reducción de la proporción de hogares en esta condición. Para este periodo, la relación de hogares pasó de 19,9\% a 15,2\% del total de hogares (CEPAL, 2007: 152).

Gráfico 2. Hogares en Asentamientos Precarios (HAP)

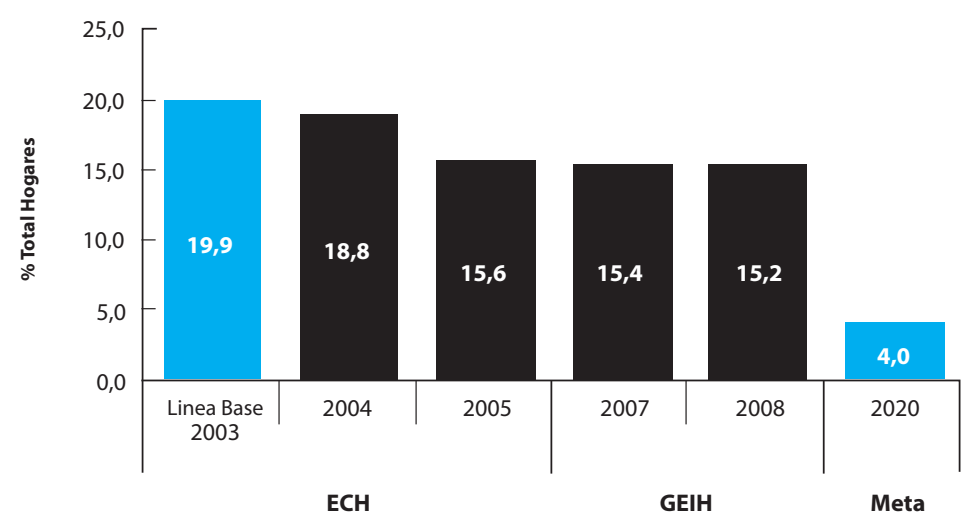

Fuente: DANE: $\mathrm{ECH}^{4} 2003$ - 2005, GEIH 2007 - 2008.

En el gráfico anterior se puede observar la evolución de los Hogares en Asentamientos Precarios desde 2003 a 2008 y la tendencia esperada a 2020. Es importante considerar que estos porcentajes hacen referencia a dos categorías de precariedad, la primera tiene que ver con condiciones mejorables de habitación y la segunda con asentamientos que no pueden mejorarse, ya sea por sus condiciones de riesgo o de infraestructura, que, por lo general, son viviendas que carecen de alcantarillado o con problemas de paredes y pisos.

\subsection{ODM en Antioquia}

El Departamento de Antioquia se integra a la política nacional de dar cumplimiento a los objetivos del milenio (ODM), y en particular, el presente

4 ECH se refiere a la Encuesta Continua de Hogares, comprendida entre los años 2001 a 2005.

5 GEIH se refiere a la Gran Encuesta Integrada de Hogares, comprendida entre los años 2007 y 2008 
escrito se ha querido centrar en el Objetivo 7 "Garantizar la sostenibilidad del medio ambiente". Antioquia ha dirigido su atención principalmente a la reducción de la pérdida de cobertura de bosques y el mejoramiento de la calidad de vida de las personas en situación de pobreza y miseria, en los subtemas de saneamiento básico y acceso al agua potable.

De acuerdo con el segundo informe nacional de seguimiento de los ODM, se ha encontrado como principal dificultad la acentuada inequidad que existe entre las regiones. Debido a esta razón, la estrategia del Gobierno colombiano para disminuir esta desigualdad es enfocar sus acciones a escala departamental y municipal, donde el mayor trabajo se requiere para conseguir las metas planteadas.

\subsection{ODM en el Oriente Antioqueño}

En el tema del medio ambiente el Oriente Antioqueño trazó su ruta ambiental hasta el año 2032 a través de un Plan de gestión ambiental regional de Cornare ${ }^{6}$ (2014), aportando como presupuesto dos billones de pesos y comprometiéndose con que para 2032 el 100\% de los municipios de la región tengan cobertura universal en saneamiento básico y agua potable, por eso se tiene como objetivo llegar a cinco municipios: Guatapé, Concepción, Alejandría, El Peñol, La Ceja y El Retiro.

Este territorio es un escenario donde se presenta la mayor cantidad de recurso boscoso del departamento, de ahí la necesidad de implementar terrenos aptos para la preservación y reforestación. Además de esto, el Oriente Antioqueño cuenta con importantes cuencas hidrográficas que lo convierten en uno de los territorios más ricos en agua del país. Estas cuencas son administradas de acuerdo con unos parámetros legales denominados POMCAS $^{7}$ y se encuentran enmarcadas en las áreas protegidas establecidas en los planes de ordenamiento territorial que se encuentran en la jurisdicción de Cornare.

En la región del Oriente Antioqueño se ha optado por agrupar a los municipios con características similares en subregiones, para facilitar la identificación de problemas y plantear posibles soluciones colectivas para el desarrollo subregional, regional y departamental. Las subregiones quedaron agrupadas así:

6 Cornare es la Corporación Autónoma Regional de los Ríos Nare y Negro. Es la principal entidad en materia de medio ambiente y desarrollo sostenible en el Oriente Antioqueño y la organización encargada de ejecutar las políticas públicas emanadas del Sistema Nacional Ambiental - SINA.

7 POMCAS: Plan de ordenamiento y manejo de cuencas. 
Cuadro 1. Subregiones del Oriente Antioqueño

\begin{tabular}{|c|c|c|c|}
\hline ALTIPLANO & BOSQUES & EMBALSES & PÁRAMO \\
\hline $\begin{array}{c}\text { Rionegro } \\
\text { La Ceja - Santuario }\end{array}$ & Granada & El Peñol & \\
Marinilla - Guarne & San Francisco & Guatapé & Abejorral \\
San Vicente & San Luis & San Rafael & Argelia \\
El Retiro- La Unión & Cocorná & San Carlos & Nariño \\
Carmen de Viboral & Puerto Triunfo & Concepción & Sonsón \\
& & Alejandría & \\
\hline
\end{tabular}

Fuente: Documento territorial de aceleración de los ODM, Departamento de Antioquia.

En estos municipios se desarrolló la metodología propuesta por el Marco de Aceleración de los ODM (MAF por sus siglas en inglés). El MAF es una herramienta para que los países desarrollen un plan de acción de aceleración, basados en ODM prioritarios. También contribuye a que los gobiernos se ocupen de la desigualdad, una de las grandes causas en el estancamiento del progreso, enfatizando las necesidades de los grupos más vulnerables. Luego del estudio e implementación de esta metodología, se identificaron las siguientes debilidades por cada subregión (PNUD, 2012: 4):

Altiplano: $\quad$ ODM 5: Salud sexual y reproductiva.

Bosques: $\quad$ ODM 1: Ingresos y empleo con énfasis en desarrollo

Embalses: $\quad$ ODM 2: Educación con calidad y pertinencia.

Páramo: $\quad$ ODM 1: Ingresos y empleo con énfasis en desarrollo

A continuación, se hará un recuento más detallado de la situación del contexto de medio ambiente y desarrollo sostenible en las subregiones del Oriente Antioqueño, apuntando al cumplimiento del séptimo objetivo de los ODM.

\section{Subregión Altiplano}

La subregión Altiplano está constituida por ocho municipios: Rionegro, La Ceja, Marinilla, El Carmen de Viboral, Guarne, El Santuario, La Unión y El Retiro. Esta subregión representa un polo de desarrollo para el Oriente Antioqueño, debido a su cercanía con la capital del departamento, aunque, por la misma razón, padece problemáticas similares a las de la ciudad como son el incremento del consumo de sustancias psicoactivas, el hurto, el embarazo en adolescentes, entre otros. Los municipios del altiplano han servido de refugio para una gran cantidad de personas desplazadas y damnificadas por causa de la guerra y la pobreza, pues en esta subregión 
encuentran mejores oportunidades laborales y mejores condiciones de vida. La subregión presenta un alto porcentaje de embarazo adolescente que oscila entre el $16 \%$ y el $24 \%$ de las mujeres del territorio (SSSA, 2009), es una cifra que ha prendido las alarmas de las administraciones locales y que ha posibilitado el desarrollo de diferentes propuestas de educación sexual y reproductiva en los diferentes municipios.

Otro elemento preocupante en la subregión tiene que ver con la cobertura de alcantarillado y saneamiento básico en los hogares, principalmente en la población rural, que según la meta nacional se pretende alcanzar un $70 \%$, pero en la subregión el porcentaje de familias que no cuentan con este servicio se encuentra entre el $9 \%$ y el $25 \%$, y es bastante preocupante esta situación, pues aquellos municipios que no pueden garantizar sistemas de saneamiento básico a las familias tienen un alto riesgo de situarse en los niveles de pobreza y miseria. Estos indicadores son el objeto del presente artículo, los cuales están enmarcados dentro de los ODM 1 y 7 y son el principal elemento de análisis para un punto de partida en las distintas subregiones.

En esta subregión se vienen adelantando programas de mejoramiento en la gestión ambiental, inicialmente se puede mencionar el caso del manejo de los residuos sólidos en los municipios de San Vicente Ferrer, El Carmen de Viboral y la Ceja, que han tenido la iniciativa de involucrar a la comunidad en el diseño del Plan General Integral de Residuos Sólidos -PGIRS-. El principal compromiso de la ciudadanía es el proceso de separación en la fuente, recolección y aprovechamiento de residuos sólidos, dentro de un marco educativo que se inserte en la comunidad y trascienda hacia las futuras generaciones. El compromiso de las secretarías de Medio Ambiente, en asocio con EPM y Cornare, es apoyar los procesos de acopio, recolección selectiva, transformación, preindustrialización y comercialización de los residuos sólidos orgánicos e inorgánicos y, por otro lado, el apoyo a la gestión técnica, operativa y administrativa para la ejecución del proyecto.

Otra iniciativa importante para resaltar en la subregión se observa en el municipio de Marinilla donde actualmente se viene ejecutando Dimensión Agroecológica y Ambiental, que consiste en la recuperación de los recursos naturales y los sistemas productivos, con miras a consolidar el proceso de distrito agrario ${ }^{8}$ que incentiva la producción agrícola y el mejoramiento

8 Se trata de una categoría especial de ordenamiento territorial estipulada en el Decreto 3600 de 2007, en el artículo 4, numeral 2, que pretende darle un uso a terrenos que deben ser mantenidos y preservados a destinación agrícola y en los que no podría autorizarse actuaciones urbanísticas. 
de la calidad de vida de los productores campesinos; sin embargo, uno de los mayores problemas con los que se enfrenta esta dimensión es el deterioro de la base productiva, que constituye básicamente la cantidad y calidad de los recursos naturales como el agua, los suelos, el bosque y la biodiversidad asociada a estos.

El principal enfoque del Plan de desarrollo en este municipio es el impulso de la soberanía y seguridad alimentaria para mitigar el bajo nivel de ingresos de la población campesina y los altos niveles de desnutrición infantil; por otro lado, se quiere recuperar las semillas y productos ancestrales que hacían parte de la base nutricional, para que de esta forma se disminuyan las dependencias a los insumos y las externalidades que incrementan los costos de producción. Otro enfoque importante tiene que ver con las estrategias de comercialización entre productores y consumidores con el fin de disminuir, en la medida de lo posible, a los intermediarios que son los que afectan el ingreso per cápita de los productores campesinos.

\section{Subregión Bosque}

La subregión Bosque está compuesta por los municipios de San Luis, San Francisco, Cocorná y Granada. Dados los índices de pobreza y miseria que presenta la región del oriente del $25 \%$ y del $6 \%{ }^{9}$ respectivamente, esta es una de las subregiones más golpeadas por el conflicto armado colombiano $y$, en consecuencia, una de las que más altos niveles de pobreza y miseria registra. El siguiente cuadro expresa la situación de acuerdo al anuario estadístico de Antioquia 2012:

Cuadro 2. Población en pobreza y miseria por NBI en la región de bosques

\begin{tabular}{|l|c|c|c|c|}
\hline \multirow{2}{*}{ Municipio } & \multicolumn{2}{|c|}{ Pobreza } & \multicolumn{2}{c|}{ Miseria } \\
\cline { 2 - 5 } & Cabecera & Resto & Cabecera & Resto \\
\hline Cocorná & 21.15 & 31.44 & 1.69 & 7.81 \\
\hline San Francisco & 41.64 & 54.60 & 14.19 & 24.35 \\
\hline San Luis & 18.52 & 45.03 & 3.33 & 17.46 \\
\hline
\end{tabular}

Fuente: anuario estadístico de Antioquia 2012

9 Según el Anuario Estadístico de Antioquia 2010, los niveles de pobreza y miseria a nivel general son del $25.46 \%$ y $5.66 \%$ respectivamente, siendo la población rural la más afectada en un 33\% de pobreza y un $9 \%$ de miseria. 
En esta subregión se concentra el $6 \%$ de la población del oriente, y actualmente tiene un nivel de urbanización del 33\%, su población se dedica en un $24 \%$ a la producción agropecuaria, el $13 \%$ a pastos y el $62 \%$ a bosques; se dio una reducción de la natalidad y un desplazamiento interno importante como consecuencia de la violencia a finales de los 90 y principios de 2000; la presencia de los grupos armados ha dejado grandes secuelas en la población civil y consecuencias en la calidad de vida de los pobladores, principalmente por los intereses geopolíticos existentes en la zona, que además de la dinámica económica expuesta anteriormente, también en esta subregión se empiezan a desarrollar importantes proyectos mineros de extracción de piedras calizas y mármoles, megaproyectos de minería, hidroeléctricas y grandes empresas que comprometen la gobernabilidad y la sostenibilidad ambiental del territorio (PNUD, 2011: 4).

El objetivo 1 se centró principalmente en la observación de los hogares que presentan inseguridad alimentaria, un factor decisivo para determinar el nivel de pobreza extrema que, según la Encuesta de Calidad de Vida de 2009 , presenta niveles del $76 \%$ para la población urbana y $85 \%$ para la población rural. Otro indicador bastante preocupante es que ninguno de los municipios de la subregión presenta información sobre los porcentajes de desnutrición global de los niños menores de 5 años, con lo cual no se puede establecer una línea de base que permita la proyección de una meta al mediano y largo plazo.

Para el objetivo 7, los principales indicadores están centrados en la información de cobertura en acueducto y alcantarillado para la población urbana y rural, siendo esta última la más afectada, dado que solo cuenta con una cobertura del $35 \%$ en acueducto y del $14 \%$ en alcantarillado. De nuevo se presenta una frágil infraestructura de saneamiento básico en la subregión y este se convierte en uno de los principales factores de miseria en el territorio.

En la subregión es prioritario incrementar los ingresos de los habitantes, principalmente de la población rural, fortalecer la seguridad alimentaria y mejorar las competencias de la población; cada uno de estos objetivos observados desde los indicadores de Necesidades Básicas Insatisfechas $\mathrm{NBI}$, estructurados en calidad de vivienda, acceso a los servicios públicos domiciliarios, educación y dependencia económica familiar.

También es importante destacar que la subregión se encuentra en un contexto muy favorable para las iniciativas que tengan relación con los recursos naturales, el bosque, el agua y su utilización en la generación hidroeléctrica. La riqueza biológica determina un importante potencial 
productivo y de desarrollo, sin embargo, para alcanzar ese potencial es necesaria la participación de los actores locales y la puesta en marcha de propuestas económicas regionales donde las personas puedan alcanzar niveles de ingreso que les permita paulatinamente disminuir los índices de pobreza.

En este contexto, algunos representantes de los actores locales, funcionarios de las distintas entidades territoriales de la subregión y representantes de organizaciones de la subregión identificaron algunas propuestas para el mejoramiento de la dependencia económica de las familias, entre estas propuestas se encuentran:

- Proyectos productivos paneleros

- Proyectos de producción de mora, cacao, fique.

- Apoyo a la piscicultura y la ganadería, mejoramiento de especies y comercialización

- Huertas caseras y proyectos de maíz y frijol para aumentar la seguridad alimentaria.

- Maderables y aprovechamiento de recursos del bosque.

El PNUD y otras organizaciones facilitadoras identificaron mayores dificultades en los proyectos de huertas caseras, proyectos productivos agrícolas y de seguridad alimentaria, con lo cual se hizo hincapié en la necesidad de fortalecer esta iniciativa. Para ello, se implementa un proyecto en el contexto subregional, denominado "Negocio inclusivo de cacao para el mejoramiento de los ingresos de 630 familias de la subregión de bosques". El proyecto es respaldado por la Compañía Nacional de Chocolates y pretende aportarle a cada familia alrededor de dos salarios mínimos mensuales.

No obstante, estas iniciativas requieren de un acompañamiento en el proceso de consolidación de la producción y en las líneas de mercadeo, que se estima en cinco años aproximadamente. Los proyectos que respaldan estas propuestas prestan asistencia durante el primer año y en el mejor de los casos un año más para la estructuración de una base social, asociación u organización campesina que permita el fortalecimiento de los actores sociales, pero a partir del segundo año el proceso decae y la asistencia institucional que un momento fue benefactora, se convierte posteriormente en un limitante para finalidad de la iniciativa. En consecuencia, para lograr los objetivos a largo plazo, las propuestas deben pensarse también en el largo plazo y desarrollar mecanismos de asesoría y acompañamiento a los productores para que se conviertan en pequeños empresarios del agro, del turismo y de otras ideas productivas que puedan desarrollarse en la subregión. 


\section{Subregión Embalses}

La subregión de Embalses está conformada por los municipios de El Peñol, Guatapé, San Rafael, San Carlos, San Vicente Ferrer, Concepción y Alejandría; cuenta con una población de 77.257 habitantes, de los cuales el $49.5 \%$ habitan el área rural y $50.5 \%$ restantes el área urbana. Embalses es la segunda subregión del Oriente Antioqueño, después del Altiplano, que menor índice de pobreza presenta, según el Anuario Estadístico de Antioquia de 2010 (PNUD, 2011: 8).

Sin embargo, esta información corresponde al índice total de todos los municipios y en ello se considera los municipios de El Peñol y Guatapé, que vienen teniendo un desarrollo económico y social importante por sus posibilidades turísticas y su fácil acceso a las vías que conectan con la subregión del Altiplano; no obstante, según el Anuario Estadístico de Antioquia 2012, los municipios restantes presentan niveles de pobreza que oscilan entre el $25 \%$ y el $45 \%$, y de miseria entre el $3 \%$ y el $9 \%$ de su población, una cifra bastante desalentadora principalmente en los municipios de San Carlos y San Rafael. Este fenómeno se explica por la cantidad de familias retornadas ${ }^{10}$ que aumentan considerablemente las necesidades básicas de la población, en contraste con la incapacidad de las administraciones para cubrir las demandas. Los niveles que más influyen en el incremento de la pobreza son los servicios públicos domiciliarios y los bajos ingresos que tienen las familias.

Los principales problemas de la subregión se enmarcan en el acceso al agua potable y al saneamiento básico. Es paradójico que uno de los territorios que más agua produce en el país tenga este tipo de inconvenientes, y ello da cuenta de la incapacidad de las administraciones locales de realizar la gestión necesaria para aumentar los niveles de satisfacción de las necesidades básicas; por otro lado, también se puede deducir la escasa organización social que se requiere para abordar las soluciones a este tipo de problemáticas.

10 Cuando se habla de familias retornadas, se hace referencia a aquellas familias que tuvieron experiencias de desplazamiento o desalojo en el territorio por los grupos armados ilegales que estuvieron en la zona y que llevaron a estas familias a migraciones transitorias o permanentes en las ciudades de Medellín y Rionegro $y$ en algunas municipios con mayores índices de desarrollo como Marinilla, El Santuario y La Ceja; no obstante y gracias a la gestión de las autoridades nacionales y regionales se han creado programas para que estas familias regresen al territorio a reclamar sus propiedades y a establecer de nuevo su modus vivendi en los municipios. 


\section{Subregión Páramo}

En la subregión de Páramos (Argelia, Nariño, Sonsón, Abejorral), se encontró que la prioridad de este grupo de municipios se centra en disminuir la pobreza y la exclusión para el logro del ODM 1: Erradicar la pobreza extrema y el hambre, resaltando la necesidad de trabajar en iniciativas subregionales que impulsen el desarrollo local con énfasis en el fortalecimiento del relevo generacional de los productores campesinos, evitar la migración a las ciudades, asociatividad y gerencia social.

El conflicto armado en esta subregión ha tenido mucha incidencia en los índices de pobreza y miseria (principalmente en Argelia y Nariño), debido a que ha provocado desplazamientos masivos, falta de garantías para desarrollar actividades sociales, además de la crisis humanitaria propia de la guerra. La dinámica económica de la región está basada fundamentalmente en la producción agropecuaria, gracias a que es un territorio con buenos suelos y microclimas que le benefician.

Gráfico 3. Indicadores de pobreza y miseria en la subregión de Páramos.

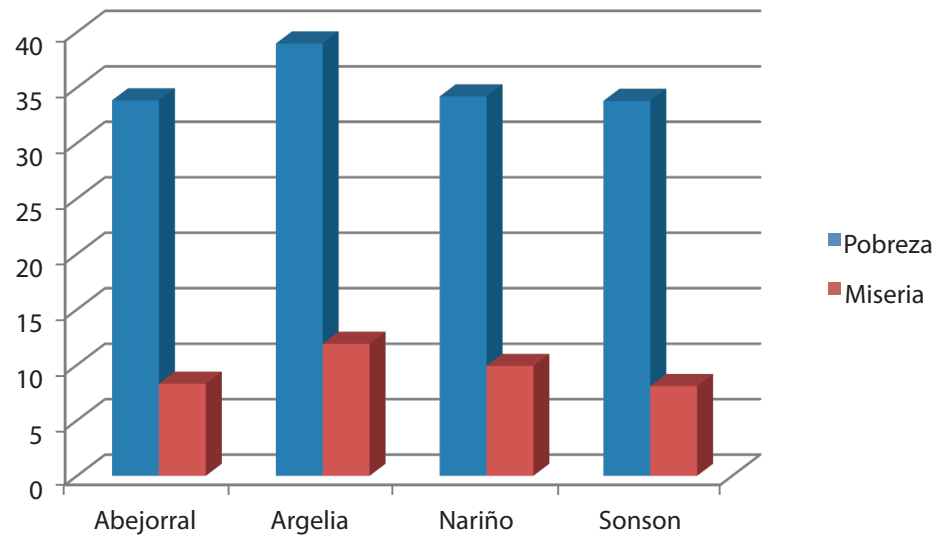

Fuente: Anuario Estadístico de Antioquia - 2010, DANE 2005, MEN: Ministerio de Educación. MIPAISOF: Programa Ministerio de Protección Social.

Al observar el gráfico anterior se puede concluir que la subregión presenta altos índices de pobreza y miseria que es el principal aspecto a trabajar en esta zona, pues, según la medición de las necesidades básicas insatisfechas, gran parte de la población tiene dificultades para acceder a vivienda, servicios sanitarios, educación básica e ingreso mínimo.

Para trabajar en la aceleración del ODM 1, la subregión tiene como prioridad enfocarse en el fortalecimiento del relevo generacional de las familias campesinas, el fortalecimiento de la cultura ciudadana y el 
desarrollo de la capacidad gerencial de proyectos para generar desarrollo y evitar la migración a las ciudades. Esta problemática se debe en gran parte a la baja productividad del sector agrícola, el uso intensivo de los suelos, los bajos niveles educativos, las vías de comunicación entre las cabeceras y las veredas; otro elemento determinante en los niveles de pobreza y miseria en el territorio es el conflicto armado, en primer lugar por la cantidad de desplazamientos y posteriormente el retorno de las familias trae como consecuencia el incremento de la cultura asistencialista generada por subsidios, ayudas humanitarias, incrementando los niveles de dependencia económica de las familias con el Estado.

Los actores locales que se han propuesto acelerar el logro de los ODM en la subregión, se han dirigido principalmente a trabajar sobre el primer objetivo. Para esto se han implementado varios programas de intervención estratégica, entre estos programas están:

- Implementación de programas alimentarios (FAO - MANÁ - PMA)

- Proyectos productivos con mujeres cabeza de hogar, campesinos y grupos organizados (lecheros, parcelación de café). Grupos organizados de producción: paneleros, Amunar (Asociación de Mujeres de Nariño) Copaco (Comité de Participación Comunitaria). Gestión de proyectos productivos Nariño

- Mejoramiento y construcción de vivienda

- Gestión de proyectos nacionales (familias guardabosques - unidos) Nariño

- Comedores escolares

- Mejoramiento vial (vías secundarias y terciarias)

- Clubes juveniles artísticos y de prevención

- Proyecto Jóvenes Exitosos Cafeteros (Aproximadamente 200 jóvenes, hombres y mujeres, entre los 15 y los 25 años).

El desarrollo de estos proyectos ha dejado ver que la mayor limitante del progreso en la subregión es la barrera cultural que abarca a estos municipios, incluida la escasa gestión política y administrativa en el acompañamiento de las propuestas socioeconómicas. La formación académica representa otra necesidad prioritaria para los jóvenes campesinos que pretenden desarrollar sus proyectos, y es importante estimular el surgimiento de líderes comunitarios para capacitarlos en actividades de participación social y cultura ciudadana.

\section{Hábitat y Desarrollo Humano}

Al analizar la situación de cada uno de los municipios que integran las distintas subregiones estudiadas, se pueden observar algunos elementos 
que coinciden en los diferentes territorios y que dan cuenta de cómo el establecimiento de las políticas públicas del Gobierno Nacional y de las administraciones locales en relación con los Objetivos de Desarrollo del Milenio no garantizan su implementación; en realidad, lo que se alcanza a visualizar es la falta de compromiso y la incompetencia de parte de las autoridades políticas y las instituciones ambientales de generar una transformación al largo plazo de las necesidades económico-sociales y ambientales en la región.

A nivel general, puede observarse cómo el problema del agua potable y el saneamiento básico es una situación presente en todas las subregiones y es uno de los principales indicadores de vulnerabilidad en las familias y un claro elemento constitutivo de pobreza y, en algunos casos, de miseria. Por esto, es necesario abordar desde una postura académica la noción de hábitat y desarrollo humano como elementos constitutivos de una aproximación a la deconstrucción de los referentes sociales asociados a la pobreza como situación de ingresos bajos, y darle una connotación que describa otros elementos que, en la mayoría de los casos, escapan al análisis de los factores que llevan a esta precaria situación.

Para explicar el hábitat es necesario entender que"el ser humano se localiza no solo en términos físicos y biológicos, sino en una multidimensionalidad natural y social, ordenada y simultánea, propia de cada sociedad, de cada territorio" (Giraldo, 2003: 43).

De ahí, la necesidad de explicar cómo el territorio que se habita y las distintas dimensiones que lo rodean determinan las condiciones vitales que puede desarrollar el ser humano, y de esta manera alterar sus condiciones sociales y ambientales. Así las cosas, una definición de hábitat que reúna los elementos que se han querido considerar en este trabajo puede ser la siguiente:

"Un hábitat digno es un elemento clave para garantizar la supervivencia de los seres humanos en condiciones de vida segura, independiente y autónoma. Las malas condiciones habitacionales son síntomas de un sistema de organización social que puede ser condicionado" (Giraldo, García, Bateman y Alonso, 2006: 28).

Es claro que las administraciones locales son permanentemente cuestionadas porque su visión política es siempre cortoplacista, y de esta manera no se piensa en los territorios como escenarios de transformación y de desarrollo al largo plazo, sino como oportunidades para el incremento del patrimonio de quienes ostentan el poder. Esta situación también demuestra la debilidad en la formación política de los habitantes de los territorios para confrontar estos intereses y darle más fuerza a la voluntad general. 
Sin embargo, al volver a la noción inicial, el hábitat es constituido por la vivienda y el entorno; el primer concepto además de la casa de habitación está constituido por el suelo, los servicios básicos, equipamiento urbano y espacio público; el segundo está constituido por las dimensiones políticas, económico-sociales, ambientales y culturales entre las que se destacan el patrimonio arquitectónico y la identidad de los territorios, y ahí también se encuentran vacíos formativos en la educación política de los individuos.

Cuando la sociedad pueda comprender la importancia de la formación política y las garantías y derechos a los que puede acceder, el mecanismo de participación en las decisiones y en las instituciones puede generar los cambios que los territorios requieren para implementar el desarrollo de los individuos y de las regiones. Según Giraldo et al. (2006), de acuerdo con la Declaración del Milenio y a los ODM, hacen referencia a la pobreza señalando a las personas, pero estas se localizan siempre en espacios concretos, en territorios y hábitats específicos, con lo cual es preciso abordar la pobreza en su dimensión territorial, y como se ha observado hasta ahora, la mayoría de las subregiones presentan situaciones similares, algunos territorios tienen situaciones más críticas como son la subregión de Páramo y Bosques para quienes va dirigida principalmente esta reflexión.

En consecuencia, el primer concepto para analizar es el de desarrollo humano que, siguiendo a Sen (2003), es el proceso de ampliación de las opciones de las personas para desarrollar sus capacidades y ponerlas al servicio de un contexto que permita, también, el desarrollo de los territorios.

En este sentido, el desarrollo humano incorpora todos los procesos y resultados que aumentan la libertad y bienestar de las personas, es decir, las garantías para la construcción de una vida saludable, acceso al conocimiento y a los recursos necesarios para permitir unas oportunidades políticas, económicas y sociales, el reconocimiento de los derechos humanos y de las libertades fundamentales.

El diagnóstico mostrado en el capítulo segundo de este artículo permite establecer unos referentes acerca de la urgencia de implementar formas de socializar las competencias ciudadanas en la comprensión de conceptos y herramientas básicas para el ejercicio de los derechos humanos y de la participación política en los escenarios locales, a fin de lograr el desarrollo humano que permita la disminución progresiva de la miseria y la pobreza en los territorios. De esta manera es preciso reconocer que "la pobreza debe entenderse no solo en términos de bajos ingresos, sino como la 
carencia de oportunidades reales para vivir una vida mínimamente adecuada" (Sen, 2003: 6).

\section{Metodología}

Se precisa conocer un escenario regional donde se puedan establecer algunas pautas de intervención sobre los elementos constitutivos de la realidad que, para el caso que nos ocupa, se refiere al mejoramiento de las condiciones de vida de la sociedad civil y la estabilidad ecológica que requiere el medio ambiente.

En este contexto regional del Oriente Antioqueño se identifican unos grupos sociales bastante diversos y con características asociadas al entorno que habitan: la subregión del Altiplano tiene una población más urbana que rural, en razón al crecimiento de la industria y el comercio en su territorio; la región de Embalses es una subregión en la que viene incrementándose la población urbana, gracias al incremento del turismo y al crecimiento comercial en las cabeceras municipales; las regiones de Bosques y Páramo son de vocación rural y lamentablemente las que muestran menores niveles de calidad de vida de sus habitantes.

La principal inquietud que intenta resolver esta investigación es saber cuál es el estado actual de la región del Oriente Antioqueño en el séptimo Objetivo del Desarrollo del Milenio. Esta pregunta lleva a los autores a considerar cada subregión en un escenario de análisis diferente, en razón a que cada territorio plantea distintos desafíos.

El propósito de la investigación es visualizar en los escenarios sociales y ambientales cómo se encuentran, a manera de diagnóstico, cada una de estas subregiones, con el fin de establecer un punto de partida para futuras intervenciones que puedan favorecer a los territorios. Se hizo una revisión bibliográfica del avance de los Objetivos del Desarrollo del Milenio en América Latina, las principales metas que componen el objetivo siete de los ODM en Colombia y posteriormente la implementación de estas metas en el Departamento de Antioquia y particularmente en la región del Oriente Antioqueño.

También se hizo un abordaje exploratorio de la información que tienen las alcaldías municipales de cada una de las subregiones con el fin de complementar los datos estadísticos que se visualizan en el desarrollo de los contenidos, sin embargo, las fuentes son bastante pobres en la mayoría de los municipios; no obstante, de los 22 municipios, ocho tienen información actualizada y relevante en el tema que nos ocupa. 


\section{Conclusiones}

En las subregiones de Embalses y Altiplano es preocupante el escaso acceso a los servicios básicos de acueducto y alcantarillado en las poblaciones rurales, que incluso con los aportes de Cornare y EPM no alcanzan a cubrir los requerimientos para garantizar el cubrimiento del $50 \%$ de la población rural del territorio en mención.

En la subregión de Bosques es prioritario incrementar los ingresos de los habitantes, principalmente de la población rural, fortalecer la seguridad alimentaria y mejorar las competencias de la población

Cada uno de estos objetivos, observados desde los indicadores de Necesidades Básicas Insatisfechas NBI, estructurados en calidad de vivienda, acceso a los servicios públicos domiciliarios, educación y dependencia económica familiar, muestran la precaria iniciativa de las autoridades locales en transformar estas realidades y la falta de participación política de sus habitantes para reclamar sus derechos.

La subregión Páramo tiene como prioridad enfocarse en el fortalecimiento del relevo generacional de las familias campesinas, el fortalecimiento de la cultura ciudadana y el desarrollo de la capacidad gerencial de proyectos para generar desarrollo y evitar la migración a las ciudades. Estas comunidades requieren el apoyo de la academia y de organizaciones no gubernamentales que puedan acompañar la gestión de estas iniciativas.

Es claro que las administraciones locales son permanentemente cuestionadas porque su visión política es siempre cortoplacista, y de esta manera no se piensa en los territorios como escenarios de transformación y de desarrollo al largo plazo, sino como oportunidades para el incremento del patrimonio de quienes ostentan el poder. Esta situación también demuestra la debilidad en la formación política de los habitantes de los territorios para confrontar estos intereses y darle más fuerza a la voluntad general.

\section{Referencias bibliográficas}

1. CEPAL. (2007). Avances en la sostenibilidad ambiental del desarrollo en América Latina y el Caribe. Disponible en Internet: http://repositorio.cepal.org/bitstream/ handle/11362/2935 /S0900696_es.pdf?sequence=1 Consultado 16.02.2016

2. COLOMBIA. Departamento Administrativo de Planeación. (2010). Anuario Estadístico de Antioquia 2009. Medellín. Gobernación de Antioquia. Disponible en Internet: http://antioquia.gov.co/PDF2/anuarios/2010/. Consultado 15.05.2016. 
3. COLOMBIA. Departamento Administrativo de Planeación. (2012). Anuario Estadístico de Antioquia 2012. Medellín. Gobernación de Antioquia. Disponible en Internet: http://www.antioquia.gov.co/antioquia-v1/organismos/planeacion/ estadísticas /anuario2012.html. Consultado 15.05.2016.

4. COLOMBIA. Departamento Administrativo de Planeación. (2005). Documento Social Conpes 91. Disponible en Internet: https://www.dnp.gov.co/ programas/ desarrollo-social/pol\%C3\%ADticas-sociales-transversales/Paginas/objetivos-dedesarrollo-del-milenio.aspx. Consultado 15.05.2016

5. COLOMBIA. Departamento Administrativo de Planeación. (2008). Los Objetivos de Desarrollo del Milenio II. Informe de seguimiento 2008. Disponible en Internet: https://www.cepal.org/MDG/noticias/paginas/2/35552/Colombia_2008.pdf. Consultado el 20.05.2016

6. COLOMBIA. Departamento Administrativo de Planeación. (2011). Documento Social Conpes 140. Disponible en Internet: https://www.dnp.gov.co/ programas/ desarrollo-social/pol\%C3\%ADticas-sociales-transversales/Paginas/objetivos-dedesarrollo-del-milenio.aspx. Consultado 15.05.2015

7. CORNARE. (2014). Informe de gestión 2014. Disponible en Internet: http://www. cornare.gov.co/component/content/article/121-planeacion-gestion-y-control/ gestion/164-informes-de-gestion. Consultado 28.06.2016

8. GIRALDO, Fabio. (2003). Hábitat y sostenibilidad en Colombia. En: Revista Colombia Ciencia y Tecnología. Vol. 21 № 2. Bogotá: Colciencias.

9. GIRALDO, Fabio; GARCÍA, Jon; BATEMAN, Alfredo y ALONSO, Andrés. (2006). Habitat y Pobreza - Los objetivos del Desarrollo del Milenio desde la ciudad. ONU - Habitat. Disponible en internet: https://books.google.es/books?hl=es\&l$\mathrm{r}=\& \mathrm{id}=\mathrm{L} 4 \mathrm{H}$ sh5QWYsQC\&oi=fnd\&pg $=\mathrm{PA} 11 \& \mathrm{dq}=$ objetivos + de + desarrollo+del+milenio+colombia\&ots=u_KDaRwi3x\&sig=Cu_yn5w6r9DDYfPoS9oHJWzT$\mathrm{BE} \# \mathrm{v}=$ onepage $\& \mathrm{q}=$ objetivos\%20de\%20desarrollo\%20del\%20milenio\%20 colombia\&f=false. Consultado 25.05.2016 ciudad. ONU - Habitat. Disponible en internet: $\quad$ https://books.google.es/books?hl=es\&lr=\&id=L4Hsh5QWYsQC\&oi=fnd\&pg=PA11\&dq=objetivos+de+desarrollo+del+milenio+colombia\&ots=u KDaRwi3x\&sig=Cu_yn5w6r9DDYfPoS9oHJWzTBE\#v=onepage\&q=objetivos $\% 20 \mathrm{de} \% 20$ desarrollo\%20del\%20milenio\%20colombia\&f=false. Consultado 25.05.2016

10. ONU. (2010). Objetivos de Desarrollo del Milenio: Avances en la sostenibilidad ambiental del desarrollo en América Latina y el Caribe. Disponible en Internet: http://www.eclac.cl/cgi-bin/getprod.asp?xml=/publicaciones/xml/6/38496/ P38496.xml\&xsl=/dmaah/. Consultado 6.6.2016

11. ONU. (2013). United Nations Statistics Division. Disponible en Internet: http://unstats.un.org/unsd/publication/SeriesF/SeriesF_78S.pdf. Consultado 30.05.2016.

12. ONU. (2015). Objetivos del Desarrollo del Milenio - Informe 2015 -. Disponible en Internet: en http://www.un.org/es/millenniumgoals/ pdf/2015/ mdgreport-2015_spanish.pdf. Consultado 17.06.2016. 
13. PNUD. (2012). Documento territorial de aceleración de los ODM - Subregión Bosques. Bogotá: Davinci Editores \& CIA S.N.C

14. PNUD. (2012). Documento territorial de aceleración de los ODM - Subregión Páramo. Bogotá: Davinci Editores \& CIA S.N.C

15. PNUD. (2012). Documento territorial de aceleración de los ODM - Subregión Altiplano. Bogotá: Davinci Editores \& CIA S.N.C

16. PNUD. (2012). Documento territorial de aceleración de los ODM - Subregión Embalses. Bogotá: Davinci Editores \& CIA S.N.C

17. PNUD. (2014). Informe de desarrollo humano 2014. Disponible en Internet: http://www.undp.org/content/undp/es/home/search.html?q=informe+sobre+desarrollo+humano+2014. Consultado 30.05.2015.

18. SEN, Amartya. (2003). La economía política de la focalización. En: Revista Comercio Exterior. Vol. 53 № 6. Ciudad de Méjico: Bancomext.

\begin{tabular}{|c|l|}
\hline $\begin{array}{c}\text { Para citar } \\
\text { este artículo: }\end{array}$ & $\begin{array}{l}\text { Osorio, Wilinthon James y Cifuentes, Juan Felipe. (2018). Evaluación del } \\
\text { estado de los Objetivos de Desarrollo del Milenio -ODM7- en la región del } \\
\text { Oriente Antioqueño. Teuken Bidikay Vol. 09 № 13. Medellín: Politécnico } \\
\text { Colombiano. Pp. 235-255 }\end{array}$ \\
\hline
\end{tabular}

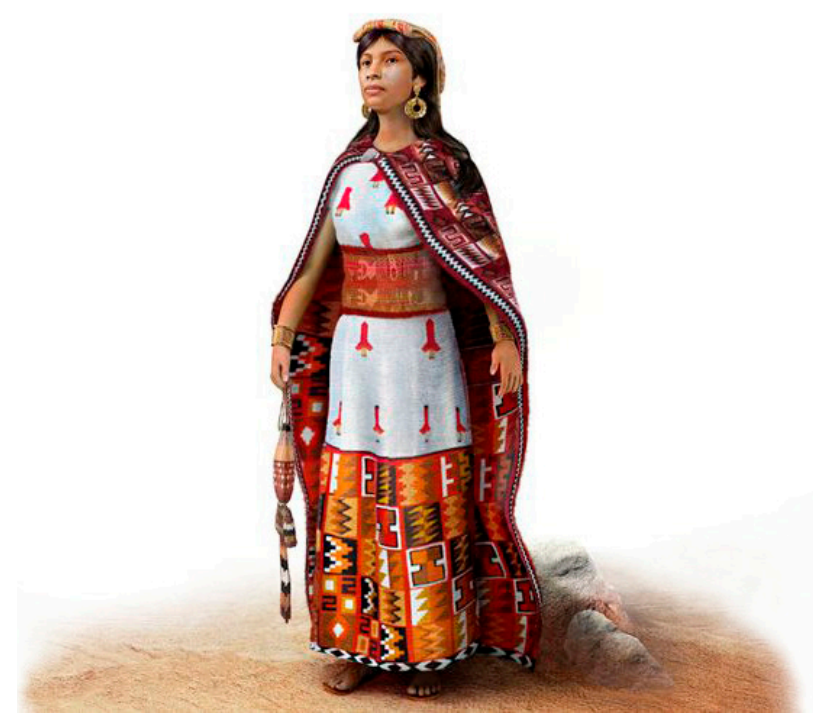




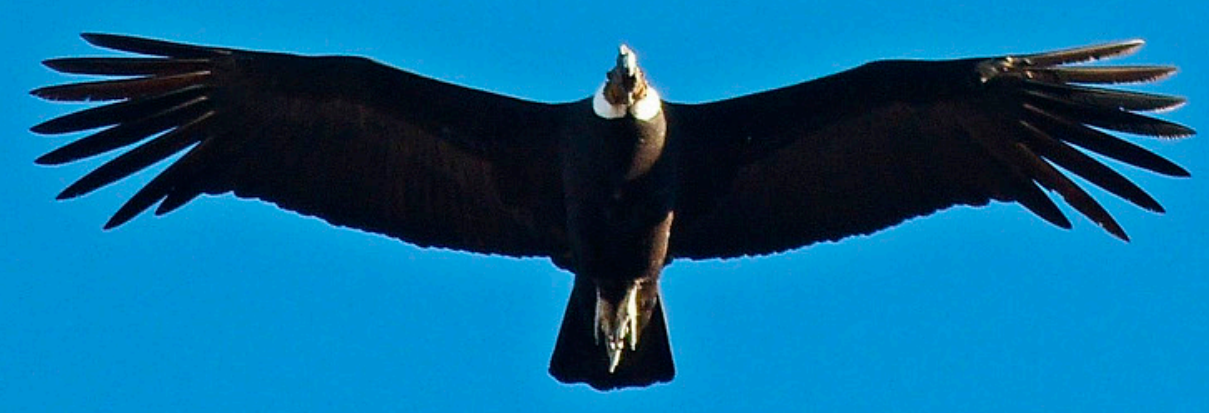

